foetus. As the prevalence of cryptorchidism in male offspring is higher when their mothers are suffering from preeclampsia or gestational diabetes, we aimed to investigate if a similar association exists between maternal SLE and cryptorchidism in the offspring.

Methods Methods: We conducted a nationwide study including all male singleton live births in Denmark from 1995 to 2016. Using the Danish nationwide population based registers, we assessed the occurrence of cryptorchidism according to prenatal disease-state of the mothers (SLE/no SLE). Cryptorchidism was assessed as both any diagnosis of cryptorchidism and diagnosis of cryptorchidism and corrective surgery. Using Cox proportional hazards models we calculated hazard ratios (HRs), accounting for varying age at time of diagnosis, and adjusting for maternal age, smoking during pregnancy, pre-gestational BMI, parity, educational level and ethnicity.

Results Among 690240 boys, 352 boys were born of mothers with pre-existing SLE. Of those, 15 (4.3\%) boys were diagnosed with cryptorchidism, and seven boys (1.9\%) underwent corrective surgery. We found an adjusted HR of 1.68 (95\% CI: 1.01, 2.78) for cryptorchidism and 1.46 (95\% CI: $0.69,3.06)$ for cryptorchidism with corrective surgery, among boys born by mothers with SLE, compared with unexposed boys. This is consistent with an increased risk of cryptorchidism among the exposed boys, even though the relatively few exposed cases limits the precision of the estimates.

Conclusions Conclusions: Boys exposed to maternal SLE appears to have higher risk of cryptorchidism, compared with unexposed boys.

Funding Source(s): Aarhus University, the Danish rheumatism society and Karen Elise Jensen foundation.

\section{7 AFRICAN AMERICAN SLE PATIENTS WITH VARIABLE DISEASE ACTIVITY REVEAL ALTERATIONS IN SIGNALING PATHWAYS AND SOLUBLE MEDIATORS THAT ARE MORE PRONOUNCED THAN EUROPEAN AMERICAN PATIENTS}

${ }^{1}$ Samantha Slight-Webb, ${ }^{1}$ Miles Smith, ${ }^{2}$ Eliza Chakravarty, ${ }^{1}$ Cristina Arriens, ${ }^{1}$ Teresa Aberle,

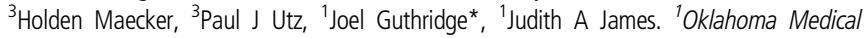
Research Foundation; ${ }^{2}$ Oklahoma Medical Research Foundation; University of Oklahoma; ${ }^{3}$ Stanford University

\subsection{6/lupus-2019-Ism.287}

Background Systemic lupus erythematosus (SLE) is an autoimmune disorder with a variable clinical presentation and periods of waxing and waning disease. Heterogeneity in SLE is influenced by genetic and non-genetic susceptibility found in different ethnicities that drive disease expression and severity. The immune pathways that contribute to heightened disease activity in lupus and immune variation by race are critical to understanding SLE disease mechanisms and outcomes.

Methods Peripheral whole blood samples of European or African American healthy controls $(\mathrm{n}=18)$ and SLE patients with either high (SLEDAI4) $(n=20)$ or low (SLEDAI $<4)(n=20)$ disease activity were stimulated for 4 min with either interferon-(IFN), PMA and ionomycin, or Toll-like receptor (TLR) ligands for either TLR4, TLR7/8 or TLR9 for phospho-protein analysis, or 24 hours for cytokine analysis of cell culture supernatants. Phenotype and phospho-protein analysis was assessed by CyTOF and cell heterogeneity was analyzed using t-SNE and manual gating. Soluble mediators were assessed using 37-plex xMAP assays and ELISA. All SLE patients met ACR classification criteria.

Abstract 287 Figure 1 African Americans SLE patients with high disease activity exhibit greater dysregulation in phospho-signaling following stimulation. Peripheral whole blood from either European or African American healthy controls, SLE patients with low disease activity (SLEDAl $<4)$ and SLE patients with high disease activity (SLEDAI $\geq 4$ ) were stimulated for 4 minutes with either no stimuli, IFN $\alpha$, PMA and ionomycin, or TLR4, TLR7/8 or TLR9 agonists. Data was collected via mass cytometry, and analyzed using Cytobank. The median 95th percentile wase used to calculate the fold change of high disease activity patients over healthy control of low disease activity patients in African American (left) or European Americans (right) in 8 cell population (B cells, CD4+ T cells, CD8+ T cells, dendritic cells (DCs), plasmacytoid DCs (pDCs), natural killer (NK) cells, monocytes (mono) and granulocytes (gran). Significant increases or decreases in phophorylation following stimulation in a particular signaling molecule are noted by a green box (decrease) or a red box (increase). The location of the dot coincides with significant differences found in a cell population (refer to legend). $p<0.05$ 
Results European American SLE patients with high disease activity were differentiated from patients with low disease activity by reduced frequencies of peripheral $\mathrm{B}$ cells, specifically naïve $B$ cells (CD27-IgD+CD24 lo) $(p=0.0101)$ and double negative $B$ cells $(C D 27-\operatorname{IgD}-)(p=0.0220)$, while African American patients with high disease activity had elevated frequencies of memory $B$ cells (CD27 $+\operatorname{IgD}$ CD $38+)$ $(\mathrm{p}<0.05)$ compared to patients with low disease activity. Several cell subsets had increased expression of activation markers during high disease activity including $B$ cells $(\mathrm{p}=0.0350)$ and plasmacytoid dendritic cells ( $\mathrm{pDCs}$ ) $(\mathrm{p}=0.0435)$ in European Americans (figure 1A), and neutrophils $(p<0.05)$, pDCs $(p=0.005)$, CD8 +T Cells $(p=0.0003)$ and NKT cells $(p=0.0033)$ in African Americans (figure 1B). Following whole blood stimulation with IFN, African American high disease activity patients were distinguished by reduced ability to activate pSTAT5 in almost all major cell populations $(\mathrm{p}<0.05)$, and pSTAT3 in monocytes $(p=0.0157)$, granulocytes $(p=0.01)$ and $B$ cells $(p=0.0409)$ compared to low disease activity patients and controls, possibly due to higher basal levels of activation (figure 1). Further, African American patients with high disease activity had significantly elevated cytokine production at baseline compared to healthy controls and European American SLE patients that translated to a reduced fold change in soluble mediators following stimulation $(\mathrm{p}<0.01)$.

Conclusions Our results support a model where race influences heightened SLE disease activity mechanisms with alterations in B cell signaling, and greater dysregulation in phospho-signaling and pro-inflammatory soluble mediators observed in African American patients.

Funding Source(s): NIH (U19AI082714, U19AI082719, U54GM104938, P30GM103510, U01AI101934)

\section{PHENOTYPIC SUBGROUPS IN SYSTEMIC LUPUS ERYTHEMATOSUS: AN EXPLORATORY CLUSTER ANALYSIS}

Amanda M Eudy*, Jennifer L Rogers, David S Pisetsky, Lisa G Criscione-Schreiber, Ravyn Njagu, Rebecca E Sadun, Kai Sun, Jayanth Doss, Megan EB Clowse. Duke University

\subsection{6/lupus-2019-Ism.288}

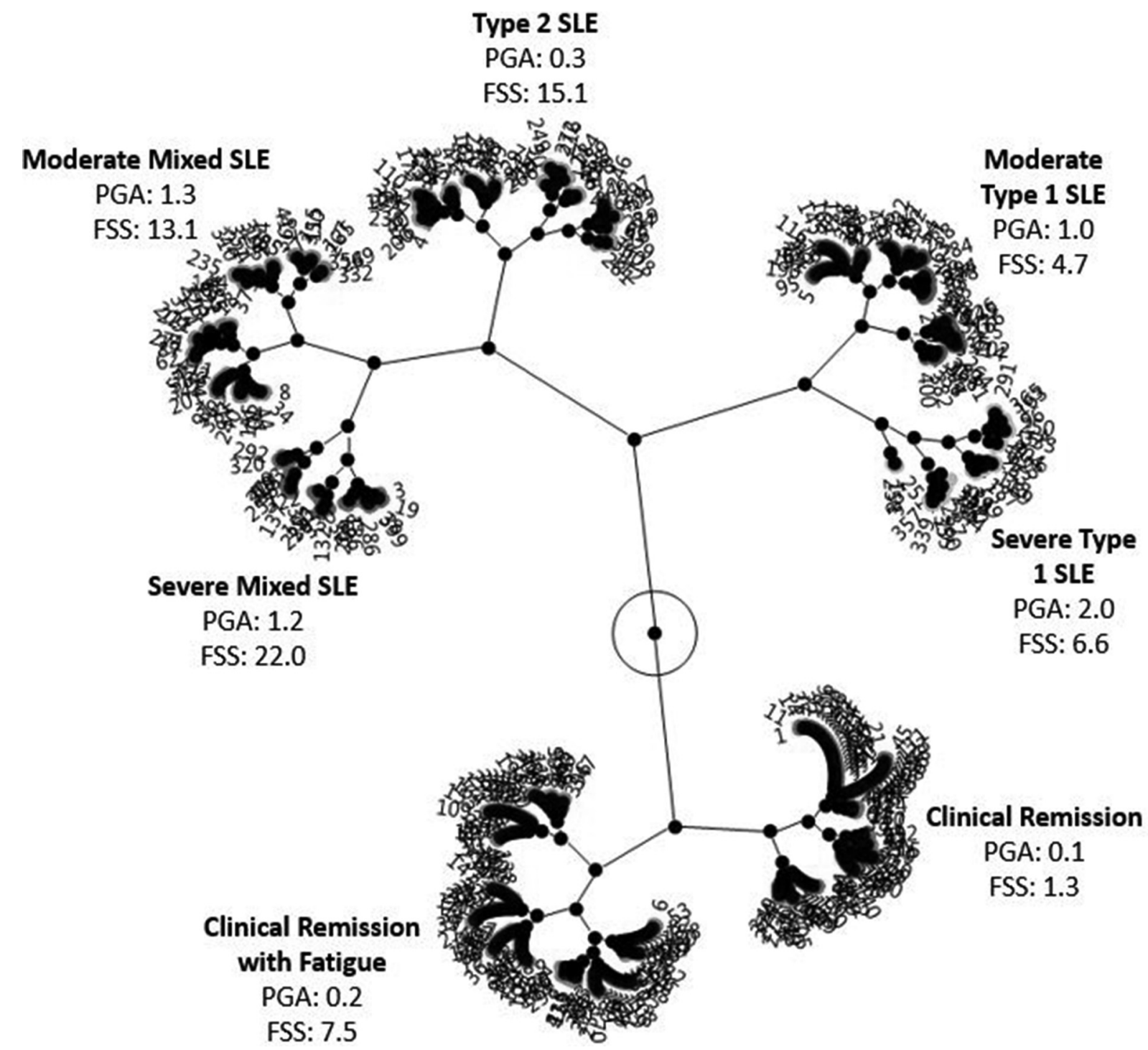

Abstract 288 Figure 1 Clustering of SLE patients by PGA and fibromyalgia severity score (FSS) 